\title{
Natura@economía
}

ISSN 2226-9479 (Versión electrónica) Website: http://revistas.lamolina.edu.pe/index.php/neu

\section{Evaluación económica de tres niveles de calcio y fósforo en dietas de cuyes (Cavia porcellus) durante la gestación y lactancia}

\author{
Economic evaluation of three levels of calcium and phosphorus in diets of guinea \\ pigs (Cavia porcellus) during pregnancy and breastfeeding
}

\author{
Giovanna Gómez O. ${ }^{1}$; Alejandrina Sotelo M.2*
}

${ }^{1}$ Universidad Nacional Agraria La Molina, Lima, Perú. asotelo@lamolina.edu.pe

Recepción: 15/02/2020; Aceptación: 15/05/2020

\section{Resumen}

El presente estudio tuvo como objetivo evaluar económicamente tres niveles de calcio y fósforo (T1: 1,0 de Ca y 0,80\% de P; T2: 1,1\% de Ca y 0,88\% de P; T3: $1,2 \%$ de Ca y 0,96\% de $\mathrm{P}$ ) en la dieta de cuyes durante las etapas de gestación y lactancia mediante la comparación de los resultados productivos al destete. Para lo cual se analizaron al término de la gestación el tamaño y peso de camada al nacimiento las ganancias de peso de las hembras reproductoras al parto, su consumo de alimento y conversión alimenticia y al finalizar la lactancia el tamaño y peso de la camada al destete, peso individual de las crías, ganancia de peso de las madres y consumo de alimento. En ese sentido, se emplearon 18 cuyes hembras en inicio de gestación, dispuestas en un diseño completo al azar (DCA), con tres tratamientos y seis repeticiones. En la primera fase los parámetros ganancia de peso y conversión alimenticia de las madres, así como el peso de la camada al nacimiento fueron influenciados significativamente $(\mathrm{P}<0,05)$ por los niveles de Ca y $\mathrm{P}$ en la dieta, presentándose los mejores resultados para el tratamiento T2. Viéndose reflejado estos resultados en la retribución económica al destete de los cuyes.

Palabras clave: calcio; fósforo; retribución; económica; cuyes; gestación; lactancia.

\begin{abstract}
The objective of this study was to economically evaluate three levels of calcium and phosphorus (T1: 1,0 Ca and 0,80\% P; T2: 1,1\% Ca and 0,88\% P; T3: 1,2\% Ca and 0,96\% P) in the guinea pig diet during the stages of gestation and lactation by comparing the productive results at weaning. For this purpose, the size and weight of the litter at birth were analyzed
\end{abstract}

Forma de citar el artículo: Gómez, G.; Sotelo, A. 2020. Evaluación económica de tres niveles de calcio y fósforo en dietas de cuyes (Cavia porcellus) durante la gestación y lactancia. Natura@economía 5(1): 27-33 (2020). 
at the end of gestation, the weight gains of the reproductive females at calving, their food intake and feed efficiency and at the end of lactation, the size and weight of the litter at weaning, individual weight of the offspring, weight gain of the mothers and feed intake of the breeding females. In this sense, 18 female guinea pigs were used at the beginning of gestation, arranged in a complete randomized design (DCA), with three treatments and six repetitions. In the gestation phase, the parameters of weight gain and nutritional conversion of the mothers, as well as the weight of the litter at birth, were significantly influenced ( $\mathrm{P}$ $<0,05)$ by the levels of $\mathrm{Ca}$ and $\mathrm{P}$ in the diet, presenting the best results for the $\mathrm{T} 2$ treatment. These results are reflected in the economic remuneration at weaning of the guinea pigs

Keywords: Calcium; phosphorus; economic; remuneration; guinea pigs; gestation; lactation.

\section{Introducción}

La creciente escasez de alimentos proteicos de origen animal ha incrementado la crianza del cuy en nuestro país impulsando diversas investigaciones en las áreas de mejoramiento genético, nutrición y manejo, permitiendo obtener características mejoradas en peso, tamaño y prolificidád. En tal sentido, el cuy constituye un producto alimenticio de alto valor nutricional, que contribuye a la seguridad alimentaria de la población generando a su vez ingresos económicos (Mamani, 2017).

Los minerales como el calcio y el fósforo son necesarios en la vida de todo animal debido a que se encuentran en gran abundancia en el cuerpo y son utilizados en diferentes procesos fisiológicos y bioquímicos (Casanueva et al., 2008).

Durante las etapas de preñez y lactancia es preciso incrementar los niveles de inclusión en la dieta, ya que si sus niveles en el plasma se reducen el organismo tiene la capacidad de adaptarse mediante la regulación hormonal y obtener estos minerales de los huesos manteniéndose la homeostasis general, como consecuencia puede producirse una descalcificación ósea, lo cual perjudicaría grandemente la producción de la siguiente camada (Caicedo et al., 2000).

Por otro lado, la crianza de cuyes genera mayor interés debido a su facilidad de crianza y abundante información técnica relacionada con su manejo, reproducción, sanidad y alimentación, es así que en la actualidad se pone de manifiesto todos los conocimientos adquiridos para disminuir los costos de producción, especialmente los de alimentación los cuales corresponden al $70 \%$ de los costos totales.

Es por ello, el interés de evaluar diferentes alternativas nutricionales que le permitan al productor mejorar su producción durante las etapas más críticas (gestación y lactancia), lo que se traduce finalmente en una mayor rentabilidad.

El objetivo del presente estudio fue evaluar económicamente tres niveles de calcio y fósforo en la dieta de cuyes durante las etapas de gestación y lactancia mediante la comparación de los resultados productivos al destete.

\section{Materiales y métodos}

El experimento fue realizado en las instalaciones del Programa de Investigación y Proyección Social en Carnes de la Facultad de Zootecnia de la Universidad Nacional Agraria la Molina con una duración de 84 días. El estudio comprendió dos etapas.

\section{Primera Etapa: "Evaluación de tres niveles de calcio y fósforo en dietas para cuyes en gestación"}

Se utilizaron 18 cuyes hembras gestantes tipo 
I (tercer parto), provenientes de la Granja de Cuyes de Cieneguilla perteneciente al Programa de Investigación y Proyección Social en Carnes de la Facultad de Zootecnia de la Universidad Nacional Agraria la Molina, las cuales fueron empadradas previamente en la misma granja con machos de igual línea y tipo evitando la consanguinidad. Se instalaron tres módulos de 10 jaulas de metal cada uno (cada módulo correspondió a un tratamiento), con un comedero de metal incorporado y un recipiente de arcilla en cada jaula. Se utilizaron tres tratamientos con la misma relación calcio: fósforo. El tratamiento $\mathrm{T} 1$ correspondiente a una dieta con $1,00 \%$ de calcio y $0,80 \%$ de fósforo total, el tratamiento $\mathrm{T} 2$ correspondiente a una dieta con $1,10 \%$ de calcio y $0,88 \%$ de fósforo total y el tratamiento T3 correspondiente a una dieta con $1,20 \%$ de calcio y $0,96 \%$ de fósforo total.

Las dietas empleadas durante el primer ensayo fueron formuladas al mínimo costo utilizando el Programa Mixit - 2 y los requerimientos nutricionales del NRC (1995). Los insumos utilizados en cada tratamiento y su correspondiente composición nutricional se observan en la Tabla 1.

El alimento balanceado fue suministrado en forma de harina durante todo el periodo de evaluación y fue ofrecido ad libitum al igual que el agua, así mismo, se realizó el análisis químico proximal de las dietas utilizadas. Los animales se pesaron individualmente al inicio y termino de gestación, y se llevó un registro del consumo de alimento diario durante este periodo.

Los controles que se midieron en cada tratamiento fueron: ganancia de peso de los cuyes hembras reproductoras al final de la gestación, consumo de alimento de los cuyes hembras reproductoras y conversión alimenticia de los cuyes hembras reproductoras. Los resultados de la progenie al parto fueron obtenidos a partir del número de crías por camada al nacimiento, peso de la camada al nacimiento, peso individual de las crías.

Tabla 1. Ingredientes y composición química de las dietas utilizadas en los periodos de gestación y lactancia

\begin{tabular}{|c|c|c|c|}
\hline Ingredientes & $\mathrm{T} 1^{1}$ & $\mathrm{~T} 2^{2}$ & $\mathrm{~T}^{3}{ }^{3}$ \\
\hline Afrecho de trigo & 56,88 & 43,38 & 41,08 \\
\hline Hominy feed & 11,10 & 20,10 & 20,60 \\
\hline Torta de soya & 17,70 & 18,40 & 19,50 \\
\hline Alfalfa molida & 11,90 & 15,20 & 15,10 \\
\hline Fosfato dicalcico & 0,41 & 1,10 & 1,60 \\
\hline $\begin{array}{l}\text { Carbonato de } \\
\text { calcio }\end{array}$ & 1,54 & 1,27 & 1,26 \\
\hline DL- Metionina & 0,10 & 0,12 & 0,41 \\
\hline Sal común & 0,32 & 0,36 & 0,36 \\
\hline $\begin{array}{l}\text { Premezcla } \\
\text { vitamínica mineral }\end{array}$ & 0,10 & 0,10 & 0,10 \\
\hline $\begin{array}{l}\text { Vitamina C, } 35 \% \\
\text { (Rovimix) }\end{array}$ & 0,02 & 0,02 & 0,02 \\
\hline Total & 100,00 & 100,00 & 100,00 \\
\hline \multicolumn{4}{|c|}{ Composición Nutricional } \\
\hline Proteína total, \% & 19,22 & 19,50 & 19,86 \\
\hline $\begin{array}{l}\text { Energía digestible, } \\
\mathrm{Mcal} / \mathrm{kg}\end{array}$ & 2,90 & 2,90 & 2,90 \\
\hline Fibra cruda, $\%$ & 12,70 & 12,80 & 12,70 \\
\hline Calcio, $\%$ & 1,01 & 1,12 & 1,21 \\
\hline Fósforo total, $\%$ & 0,80 & 0,88 & 0,96 \\
\hline
\end{tabular}

Para el análisis estadístico se utilizó un diseño completamente al azar (DCA), con tres tratamientos y 6 observaciones por tratamiento; se empleó el análisis de variancia para determinar las diferencias significativas entre los tratamientos y la prueba de Duncan (Calzada, 1982) para determinar la comparación de medias de los tratamientos en los parámetros evaluados. 
Segunda Etapa: "Evaluación de tres niveles de calcio y fósforo en dietas para cuyes en lactancia"

La segunda etapa se inició en el momento del parto de las reproductoras y culminó con el destete de las crías teniendo una duración de 14 días, para lo cual se utilizaron los mismos animales de la primera parte del experimento. Se evaluaron a las madres y a sus crías hasta el momento del destete a los 14 días, manteniéndose a las crías en las mismas jaulas. Registrándose los siguientes datos: Registros de la progenie: peso de la camada al destete, número de crías por camada al destete, peso individual de las crías al destete. Registros de la madre: consumo de alimento.

Esta segunda etapa se ajustó también a un Diseño Completamente al Azar, para tres tratamientos con seis repeticiones cada uno. Se realizó el análisis de variancia para determinar las diferencias significativas entre los tratamientos y la prueba de Duncan (Calzada, 1982) para determinar la comparación de medias de los tratamientos.

\section{Resultados y discusiones}

Primera Etapa: "Evaluación de tres niveles de calcio y fósforo en dietas para cuyes en gestación"

Las ganancias de peso revelaron diferencias estadísticas entre los tratamientos $(\mathrm{p}<0,05)$, siendo el tratamiento $\mathrm{T} 2$ el que mostró mayor incremento de peso respecto a los tratamientos T3 y T1 como se observa en la Tabla 2.

El comportamiento de las hembras en relación al consumo de alimento de materia seca total durante el periodo de gestación fue similar, no presentando diferencias estadísticas $(p>0,05)$ entre los tratamientos como se muestra en la Tabla 2.
Tabla 2. Parámetros productivos evaluados en las hembras reproductoras durante la etapa de gestación

\begin{tabular}{lccc}
\hline \multicolumn{1}{c}{ Parámetros } & \multicolumn{3}{c}{ Tratamientos } \\
\cline { 2 - 4 } & T1 & T2 & T3 \\
\hline $\begin{array}{l}\text { Número de } \\
\text { animales }\end{array}$ & 6 & 6 & 6 \\
$\begin{array}{l}\text { Peso de } \\
\text { apareamiento, } \\
\text { g }\end{array}$ & 1273,00 a & 1270,00 a & 1265,00 a \\
$\begin{array}{l}\text { Peso final de } \\
\text { gestación, g }\end{array}$ & 1757,00 & 2030,00 & 2015,00 \\
$\begin{array}{l}\text { Ganancia de } \\
\text { peso, g }\end{array}$ & 483,83 a & 760,23 b & 496,52 a \\
$\begin{array}{l}\text { Consumo de } \\
\text { alimento, g }\end{array}$ & 3340,32 & 3433,92 & 3371,52 \\
$\begin{array}{l}\text { Número de } \\
\text { días }\end{array}$ & 48 & 48 & 48 \\
$\begin{array}{l}\text { Ganancia de } \\
\text { peso/día, g }\end{array}$ & 10,08 & 15,84 & 10,34 \\
$\begin{array}{l}\text { Consumo de } \\
\text { alimento } / 1 \\
\text { día, g }\end{array}$ & $69,59 \mathrm{a}$ & $71,54 \mathrm{a}$ & $70,24 \mathrm{aa}$ \\
$\begin{array}{l}\text { Conversión } \\
\text { alimenticia }\end{array}$ & $6,90 \mathrm{a}$ & $4,51 \mathrm{~b}$ & $6,79 \mathrm{a}$ \\
$\begin{array}{l}\text { a, b; letras diferentes en cada fila indican diferencia } \\
\text { estadística (P<0,05) } \\
\text { 1Consumo de alimento en base a materia seca }\end{array}$
\end{tabular}

Las conversiones alimenticias revelaron diferencias estadísticas $(p<0,05)$ en el análisis de variancia entre los tratamientos. Siendo el tratamiento T2 el que obtuvo un mejor valor a la prueba de Duncan en comparación con los demás tratamientos T1 y T3, notándose que al incrementarse los niveles de minerales calcio y fósforo en la dieta la conversión alimenticia baja de igual forma como se ha visto en el consumo de alimento. Estos resultados concuerdan con los reportados por Caicedo et al. (1983), al evaluar diferentes niveles de calcio y fósforo para cuyes gestantes, donde se obtuvieron buenos valores de conversión alimenticia para niveles de calcio $1,08 \%$ y fósforo $0,68 \%$ como consecuencia de haber obtenido el mejor incremento de peso. 
El tamaño promedio de las camadas al nacimiento se muestra en el cuadro 3, observándose que no existen diferencias estadísticas $(\mathrm{p}>0,05)$ entre los distintos tratamientos.

En la Tabla 3 se presentan los resultados obtenidos de los pesos de las camadas al nacimiento, donde se observa que existe diferencias estadísticas $(\mathrm{p}<0,05)$ entre los tratamientos, siendo el tratamiento $\mathrm{T} 2$ el de mayor valor, tanto el tratamiento $\mathrm{T} 3$ como el tratamiento $\mathrm{T} 1$ presentaron pesos similares. De igual forma, Vergara (2008) describe pesos de 558,00 g para camadas de tres crías y $388,00 \mathrm{~g}$ para camadas de dos crías, usando solo alimento balanceado integral durante la gestación de las madres.

Tabla 3. Resultados de la progenie al parto

\begin{tabular}{lccc}
\hline \multirow{2}{*}{ Parámetros } & \multicolumn{3}{c}{ Tratamientos } \\
\cline { 2 - 4 } & T1 & T2 & T3 \\
\hline $\begin{array}{l}\text { Número de } \\
\text { crias por } \\
\text { camada al } \\
\text { nacimiento }\end{array}$ & $2,00 \mathrm{a}$ & $3,33 \mathrm{a}$ & $2,16 \mathrm{a}$ \\
$\begin{array}{l}\text { Peso de la } \\
\text { camada al } \\
\text { nacimiento, } \mathrm{l}\end{array}$ & $354,00 \mathrm{a}$ & $576,78 \mathrm{~b}$ & $363,42 \mathrm{a}$ \\
$\begin{array}{l}\text { Peso de la } \\
\text { camada al } \\
\text { nacimiento, g }\end{array}$ & $168,03 \mathrm{a}$ & $182,58 \mathrm{a}$ & $168,88 \mathrm{a}$ \\
$\begin{array}{l}\mathrm{a} \text { a b; letras diferentes en cada fila indican diferencia } \\
\text { estadística }(\mathrm{P}<0,05)\end{array}$
\end{tabular}

El número de crías al parto influyó en el peso de la camada al nacimiento, siendo el tratamiento T2 el que obtuvo un mayor número de crías y el tratamiento $\mathrm{T} 1$ el de menor valor. Pudiéndose deducir que con relación a este parámetro los rendimientos son adecuados hasta un nivel de calcio y fósforo de $1,1 \%$ y $0,88 \%$, respectivamente.

\section{Segunda Etapa: "Evaluación de tres niveles de calcio y fósforo en dietas para cuyes en lactancia"}

En la Tabla 4 se puede observar que los tratamientos tuvieron un comportamiento semejante en los parámetros evaluados.

Tabla 4. Rendimiento de cuyes hembras en lactancia

\begin{tabular}{|c|c|c|c|}
\hline \multirow{2}{*}{ Parámetros } & \multicolumn{3}{|c|}{ Tratamientos } \\
\hline & $\mathrm{T} 1$ & $\mathrm{~T} 2$ & T3 \\
\hline $\begin{array}{l}\text { Número de } \\
\text { animales }\end{array}$ & 6 & 6 & 6 \\
\hline Peso al parto, $g$ & 1470,66 & 1461,33 & 1473,00 \\
\hline $\begin{array}{l}\text { Peso al destete, } \\
\mathrm{g}\end{array}$ & 1410,83 & 1338,33 & 1372,83 \\
\hline $\begin{array}{l}\text { Aumento de } \\
\text { peso.g }\end{array}$ & $-59,83 \mathrm{a}$ & $-123,00 \mathrm{a}$ & $-100,17 \mathrm{a}$ \\
\hline $\begin{array}{l}\text { Aumento de } \\
\text { peso/día, g }\end{array}$ & $-4,27$ & $-8,78$ & $-7,15$ \\
\hline $\begin{array}{l}\text { Consumo de } \\
\text { alimento 1, g }\end{array}$ & 1178,82 & 1177,05 & 1179,42 \\
\hline $\begin{array}{l}\text { Consumo de } \\
\text { alimento2/día, g }\end{array}$ & $84,20 \mathrm{a}$ & $84,07 \mathrm{a}$ & $84,24 \mathrm{a}$ \\
\hline \multicolumn{4}{|c|}{$\begin{array}{l}\text { a, b; letras diferentes en cada fila indican diferencia } \\
\text { estadística }(\mathrm{P}<0,05)\end{array}$} \\
\hline \multicolumn{4}{|c|}{$\begin{array}{l}{ }^{1,2} \text { Consumo promedio en base seca de la madre más sus } \\
\text { crías }\end{array}$} \\
\hline
\end{tabular}

El número de crías por camada al destete se muestra en la Tabla 5, donde se observan que los tratamientos T1, T2 y T3 no presentaron diferencias estadísticas entre sí $(p>0,05)$. Como se indica en dicho cuadro los tratamientos T1 y T3 no variaron en su tamaño desde el nacimiento hasta conseguir el destete.

El peso de la camada al destete se indica en la Tabla 5, no se presentaron diferencias estadísticas $(p>0,05)$ entre los tratamientos para dicho parámetro. Al respecto, Caicedo et al. (1983), señala que el número de gazapos destetados influye en el peso total de la camada. Resultados obtenidos por Vergara (2008), al evaluar un alimento balanceado integral en dietas de inicio indican un resultado similar en el peso de la camada al destete. Sobre el particular Ordoñez (1997) indica que las crías duplican su peso durante la lactancia llegando a incrementar hasta en un $95 \%$ de su peso al nacimiento. 
Tabla 5. Resultado de la progenie al destete

\begin{tabular}{|c|c|c|c|}
\hline \multirow{2}{*}{ Parámetros } & \multicolumn{3}{|c|}{ Tratamientos } \\
\hline & $\mathrm{T} 1$ & $\mathrm{~T} 2$ & $\mathrm{~T} 3$ \\
\hline $\begin{array}{l}\text { Número de crías } \\
\text { por camada al } \\
\text { nacimiento }\end{array}$ & 2,00 & 3,33 & 2,16 \\
\hline $\begin{array}{l}\text { Número de crías } \\
\text { por camada al } \\
\text { destete }\end{array}$ & $2,00 \mathrm{a}$ & $3,00 \mathrm{a}$ & $2,16 \mathrm{a}$ \\
\hline $\begin{array}{l}\text { Peso de la camada } \\
\text { al nacimiento, g }\end{array}$ & 354,06 & 576,78 & 363,42 \\
\hline $\begin{array}{l}\text { Peso de la camada } \\
\text { al destete, g }\end{array}$ & $782,00 \mathrm{a}$ & $977,32 \mathrm{a}$ & 819,62 a \\
\hline $\begin{array}{l}\text { Peso individual al } \\
\text { nacimiento, } g\end{array}$ & 168,03 & 182,58 & 168,88 \\
\hline $\begin{array}{l}\text { Peso individual al } \\
\text { destete, } g\end{array}$ & 308,67 a & $276,51 \mathrm{a}$ & $307,02 \mathrm{a}$ \\
\hline $\begin{array}{l}\% \text { de mortalidad al } \\
\text { destete }\end{array}$ & $0 \%$ & $6,6 \%$ & $0 \%$ \\
\hline
\end{tabular}

Respecto al peso individual de las crías no hubo diferencias estadísticas $(\mathrm{p}>0,05)$, pero si se pudo observar que el tratamiento T1 superó ligeramente a los tratamientos restantes probablemente debido al menor número de crías destetadas, de igual forma el tratamiento T2 obtuvo un peso menor en comparación a los T1 y T3.

Por otro lado, el experimento demuestra la importancia de suministrar un adecuado balance de nutrientes en la fase de lactancia, debido a que es posible una recuperación total del peso en camadas que nacieron con pesos bajos, e inclusive superar en pesos a aquellos animales que al nacimiento tuvieron pesos mayores (Caicedo et al., 1983).

En la Tabla 6 se detalla la retribución económica por kilogramo de carne de cuy producida por tratamiento hasta la etapa de destete. Los cuyes reproductores alimentados con el tratamiento T2 presentaron una mejor retribución económica por kilogramo de cuy producido, superando a los tratamientos $\mathrm{T} 1$ y T3.
Tabla 6. Retribución económica

\begin{tabular}{lccc}
\hline \multirow{2}{*}{\multicolumn{1}{c}{ Parámetros }} & \multicolumn{3}{c}{ Tratamientos } \\
\cline { 2 - 4 } & $\mathrm{T} 1$ & $\mathrm{~T} 2$ & $\mathrm{~T} 3$ \\
\hline Nro. de crías/camada & 2,00 & 3,00 & 2,16 \\
Peso destete (kg) & 0,31 & 0,28 & 0,31 \\
Precio kg de cuy (S/.) & 25,00 & 25,00 & 25,00 \\
Ingreso bruto/ kg (S/.) & 15,50 & 21,00 & 16,74 \\
Egresos & & & \\
$\begin{array}{l}\text { Costo de alimentación } \\
\text { (S/.) }\end{array}$ & 1,19 & 1,25 & 1,30 \\
$\begin{array}{l}\text { Consumo de alimento } \\
\text { (kg) }\end{array}$ & 4,52 & 4,16 & 4,55 \\
$\begin{array}{l}\text { Costo de crianza (S/.) } \\
\text { Costo total/kg (S/.) }\end{array}$ & 1,00 & 1,00 & 1,00 \\
$\begin{array}{l}\text { Retribución económica (S/.) } \\
\text { Por kg de peso vivo }\end{array}$ & 10,12 & 15,24 & 5,92 \\
\hline
\end{tabular}

\section{Conclusiones}

Los mejores rendimientos durante la etapa de gestación se presentaron con el tratamiento T2 para los parámetros ganancia de peso y conversión alimenticia de las gestantes, de igual forma el tratamiento T2 obtuvo un mejor resultado al evaluar el peso total de la camada al parto.

La mejor retribución económica por $\mathrm{kg}$ de peso vivo de cuy la obtuvo el tratamiento T2.

\section{Literatura citada}

Caicedo, A.; Muñoz, J.; Caicedo, V. 1983. Evaluación de diferentes niveles de calcio y fósforo, en los periodos de gestación y lactancia de cuyes. Tesis de Ingeniero Agrónomo: Pasto. Colombia. Universidad de Nariño.

Caicedo, A. 2000. Experiencias investigativas en producción de cuyes. Contribución al desarrollo tecnológico de la especie: Pasto, Colombia. Universidad de Nariño.

Calzada, B. 1982. Métodos estadísticos para la investigación. 5ta edición. Lima: Editorial Jurídica.

Casanueva, E.; Kaufer, M.; Pérez, A.; 
Arroyo, P. 2008. Nutrilogía Médica. 3ra edición. México: Editorial Médica Panamericana.

Mamani, L. 2017. Caracterización económica de la crianza de cuyes de la red distrital de criadores de cuy en la microcuenca del distrito de Pitumarca - Canchis - Cusco. Tesis de Ingeniero Zootecnista. Cusco. Perú: Universidad San Antonio Abad del Cusco.192p.

NRC [National Research Council]. 1995. Nutrient requirements of laboratory animals. No. 9. Washington, D.C.

Ordoñez, R. 1997. Efecto de dos niveles de proteína y fibra cruda en el alimento de cuyes (Cavia Porcellus) en la lactación y crecimiento. Tesis de Ingeniero Zootecnista. Lima. Perú. Universidad Nacional Agraria la Molina. 84p.

Vergara, V. 2008. Simposio: Avances sobre producción de cuyes en el Perú. En XXXI Reunión Científica Anual de la Asociación Peruana de Producción Animal (APPA). Lima: Universidad Nacional Agraria la Molina. 